\title{
Autophagy in Yeast: A Review of the Molecular Machinery
}

\author{
Wei-Pang Huang and Daniel J. Klionsky* \\ Departments of Molecular, Cellular and Developmental Biology and Biological Chemistry, and the Life \\ Sciences Institute, University of Michigan, Ann Arbor, MI 48109-1048, USA
}

\begin{abstract}
Autophagy is a membrane trafficking mechanism that delivers cytoplasmic cargo to the vacuole/ lysosome for degradation and recycling. In addition to non-specific bulk cytosol, selective cargoes, such as peroxisomes, are sorted for autophagic transport under specific physiological conditions. In a nutrient-rich growth environment, many of the autophagic components are recruited for executing a biosynthetic trafficking process, the cytoplasm to vacuole targeting (Cvt) pathway, that transports the resident hydrolases aminopeptidase $I$ and $\alpha$ mannosidase to the vacuole in Saccharomyces cerevisiae. Recent studies have identified pathway-specific components that are necessary to divert a protein kinase and a lipid kinase complex to regulate the conversion between the Cvt pathway and autophagy. Downstream of these proteins, the general machinery for transport vesicle formation involves two novel conjugation systems and a putative membrane protein complex. Completed vesicles are targeted to, and fuse with, the vacuole under the control of machinery shared with other vacuolar trafficking pathways. Inside the vacuole, a potential lipase and several proteases are responsible for the final steps of vesicle breakdown, precursor enzyme processing and substrate turnover. In this review, we discuss the most recent developments in yeast autophagy and point out the challenges we face in the future.
\end{abstract}

Key words: aminopeptidase I (Ape1)/autophagy/cytoplasm to vacuole targeting (Cvt) pathway/pexophagy

Eukaryotic cells have two major avenues for the degradation of proteins and organelles, the proteasome and the vacuole/lysosome. The proteasome and vacuole/lysosome complement each other to achieve the task of turning over a spectrum of protein and organelle substrates under a variety of physiological conditions. The proteasome is a compartmentalized protease machine with its enzymatic activities embedded inside a central proteinaceous chamber (Voges et al., 1999). This chamber opens to its surrounding cytoplasm or nucleoplasm through aqueous channels. As a result, the degradative capacity of the proteasome is confined to partially denatured protein substrates that can access its proteolytic subunits. Cytoplasmic and nuclear short-lived proteins and misfolded proteins destined for proteasomemediated degradation are tagged with ubiquitin signals

*To whom correspondence should be addressed: Daniel J. Klionsky, Departments of Molecular, Cellular and Developmental Biology and Biological Chemistry, and the Life Sciences Institute, University of Michigan, Ann Arbor, MI 48109-1048, USA.

Tel: +1-734-615-6556, Fax: +1-734-647-0884

E-mail: klionsky@umich.edu

Abbreviations: Ams1, $\alpha$-mannosidase; Ape1, aminopeptidase I; Cvt, cytoplasm to vacuole targeting; PAS, pre-autophagosomal structure; PP2A, protein phosphatase 2A; prApe1, precursor aminopeptidase I; PtdIns, phosphatidylinositol; rER, rough endoplasmic reticulum.
(Ulrich, 2002), adding an additional regulatory mechanism for proteasome recognition. While the architectural designs of proteasomes ensure efficient processing kinetics for soluble proteins, they limit the ability for proteasomes to act on membrane proteins and protein complexes, and completely prevent their access to membrane enclosed compartments. To this end, the vacuole of yeasts and the lysosome of mammalian cells are suitable for degrading larger and more complex substrates including protein complexes and organelles (Teter and Klionsky, 2000). The vacuole/lysosome maintains a panoply of enzymes contained within a limiting membrane that segregates these hydrolases from the surrounding cytoplasm. This compartmentalization circumvents the need for substrate selection based on size exclusion employed by proteasomes, but raises new challenges for substrate delivery. Every cytosolic molecule entering the vacuole/lysosome, including hydrolases and substrates, has to pass through the barrier of the limiting membrane. Accordingly, multiple membrane-trafficking pathways are dedicated to guarantee the normal function of the vacuole/ lysosome (Wendland et al., 1998).

The majority of vacuolar hydrolases are delivered to this organelle through the compartments of the early secretory pathway and are sorted away from secretory proteins at the 
trans-Golgi Network where they are recognized due to the presence of specific vacuolar targeting signals (Bryant and Stevens, 1998). At least three different routes-the carboxypeptidase Y (CPY, Prc1) pathway, the alkaline phosphatase (ALP, Pho8) pathway, and the multivesicular body (MVB) pathway followed by carboxypeptidase $S$ (Cps1)-for vacuolar enzyme delivery from the trans-Golgi Network to the vacuole have been identified. One common aspect of these vacuolar biosynthetic trafficking pathways is that their cargoes are all translocated co-translationally at the rough endoplasmic reticulum (rER). The rER is specially equipped for translocating proteins kept in an unfolded or partially folded state (Oliver et al., 1995). The substrates for vacuolar degradative activity are primarily fully folded proteins and even organelles in some cases, which necessitate specific mechanisms for entering the lumen of the vacuole (Teter and Klionsky, 1999). For substrates that originate from the extracellular environment, or the topologically equivalent domain in the lumen of intracellular organelles, relatively simple mechanisms involving transport vesicles can mediate delivery. In contrast, other substrates must be moved from their cytosolic localization to the vacuole lumen, which corresponds to an extracellular milieu. From a mechanistic point of view, two general processes can accomplish this change in subcellular topology. Substrates can be translocated directly into the lumen at the vacuole/ lysosome surface. This process is seen with chaperonemediated autophagy of mammalian proteins that contain a consensus KFERQ-sequence tag (Dice, 1992). A similar process may occur in yeast (Horst et al., 1999). In addition, cytosolic proteins may be imported into cytosolic transport vesicles that subsequently fuse with the vacuole membrane, as occurs in the vacuolar import and degradation (Vid) pathway-mediated turnover of fructose-1,6-bisphosphatase (FBPase) in Saccharomyces cerevisiae (Huang and Chiang, 1997). In general, these pathways require protein chaperones presumably to partially unfold the substrates (Agarraberes and Dice, 2001; Brown et al., 2000). The specific mechanism(s) by which the protein substrates translocate across the vacuole/lysosome or vesicle membrane remains to be determined. Alternatively, mechanisms with doublemembrane transport intermediates, such as macroautophagy, avoid the process of membrane translocation, and are therefore especially suitable for delivery of protein complexes and organelles (Kim et al., 2000). In this review, we discuss the mechanism of macroautophagy and give special emphasis to the most recent advances in macroautophagy studies in Saccharomyces cerevisiae.

\section{Autophagy and related vacuolar trafficking pathways}

Autophagy is a membrane trafficking process that transports bulk cytoplasm and sometimes entire organelles to the vacuole for recycling in response to nutrient starvation or during specific physiological conditions, such as hormonal stimuli in mammalian cells (Klionsky and Emr, 2000). Autophagy has two major forms, microautophagy and macroautophagy (Fig. 1). Microautophagy operates by protruding or invaginating a portion of the vacuolar membrane to engulf cytosol or organelles. Only limited knowledge is available about microautophagy, which has been best characterized for the degradation of peroxisomes (Sakai et al., 1998; Yuan et al., 1997). Accordingly, this review concentrates on macroautophagy and its related pathways. Macroautophagy involves the de novo formation of a sequestering vesicle in the cytosol (Noda et al., 2002). The transport vesicles formed for the macroautophagy pathway, autophagosomes, employ a double-membrane mechanism. A consequence of the double-membrane transport vesicles is that cargo ends up with an opposite cellular topology. Autophagosomes completely segregate their cargo, a portion of cytosol and/or organelles, from surrounding cytoplasm, and upon completion are targeted to the vacuole. Fusion of the outer autophagosome membrane with the vacuole releases the inner membrane vesicle, called an autophagic body, into the vacuolar lumen. Vacuolar hydrolases disintegrate the membrane of autophagic bodies and process their cargo to building blocks for reuse, which marks the final stage of macroautophagic vacuolar trafficking. A complete progression of autophagy events delivers cytosolic substrates to the vacuolar lumen without their having to directly pass through a membrane barrier. While autophagy is known as an inducible catabolic process, studies in the past decade have uncovered a biosynthetic vacuolar trafficking pathway in the yeast Saccharomyces cerevisiae, termed the cytoplasm to vacuole targeting (Cvt) pathway, that operates through a mechanism that is similar to macroautophagy.

The first evidence indicating an alternative biosynthetic vacuolar trafficking pathway came from a study of the resident vacuolar hydrolase $\alpha$-mannosidase (Ams1; Yoshihisa and Anraku, 1990). More conclusive information became available with the discovery that the precursor form of vacuolar aminopeptidase I (Ape1) was delivered independent of the secretory pathway (Klionsky et al., 1992). The $61 \mathrm{kDa}$ aminopeptidase I precursor (prApe1) is synthesized in the cytosol. Rapid oligomerization leads to homododecameric units as the major initial cytosolic form of prApe1 (Kim et al., 1997). Immunoelectron microscopy studies further identified a cytosolic electron-dense structure, called the Cvt complex, containing multiple prApe1 dodecamers (Baba et al., 1997; Scott et al., 1997). The Cvt complex is usually seen associated with double-membrane cisternae. The identity of the membrane structure is not clear, but it is considered to be an intermediate structure in the process of completely enclosing the Cvt complex to eventually make a double-membrane vesicle, the Cvt vesicle. As with autophagosomes, completely formed $\mathrm{Cvt}$ vesicles are targeted to and fuse with the vacuole, releasing the inner membrane vesicles, called Cvt bodies, into the 


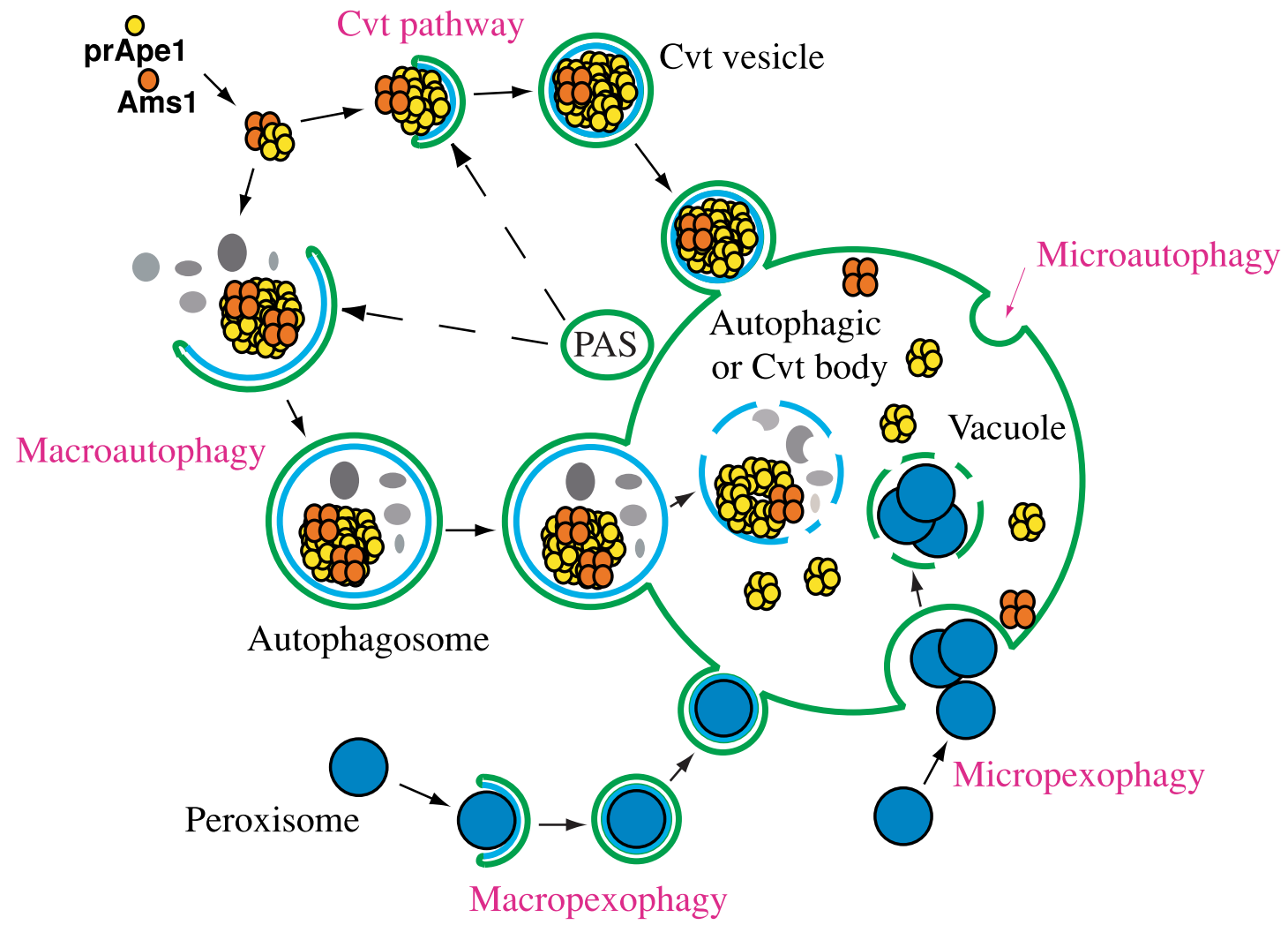

Fig. 1. Morphology of the Cvt pathway, autophagy and pexophagy. The pre-autophagosomal structure (PAS) is the presumed sight of Cvt vesicle and autophagosome formation and/or the origin of the donor membrane. Cvt vesicles form under vegetative conditions while autophagosomes form by macroautophagy during starvation. Macropexophagy occurs following a shift from peroxisome-inducing conditions to a preferred carbon source. Microautophagy and micropexophagy involve uptake directly at the vacuole surface. See text for additional details.

vacuolar lumen. Vacuolar hydrolases degrade the Cvt body membrane and process prApe 1 to its $50 \mathrm{kDa}$ mature form. The overall delivery process of prApe1 by the Cvt pathway is essentially similar to the macroautophagy pathway. In fact, the Cvt complex was found inside of autophagosomes of starved yeast cells (Baba et al., 1997), indicating a possible cognate relation between the Cvt and macroautophagy pathways. For simplicity, macroautophagy will be referred to as autophagy in the rest of this review.

A third vacuolar transport mechanism related to the Cvt and autophagy pathways is pexophagy, selective autophagy of peroxisomes (Kim and Klionsky, 2000). Depending on environmental conditions and the organism, pexophagy operates through either a micropexophagy or macropexophagy mechanism (Fig. 1). These two modes of pexophagy have similar morphologies to their corresponding types of autophagy, except that the cargo is peroxisomes.

\section{Comparison between the Cvt pathway and autophagy}

In addition to morphological similarities, genetic analyses also confirmed the close relationship between the Cvt and autophagy pathways. A screen for defective prApe1 import under vegetative growth conditions allowed the isolation of a set of $c v t$ mutants (Harding et al., 1995). The $c v t$ mutants partially overlapped with autophagy deficient mutants, apg and aut (Harding et al., 1996; Scott et al., 1996). The majority of non-overlapping strains in the three mutant collections also show defects for the other pathway. Based on what we now know regarding the similar mechanisms used for vacuolar delivery, it is not surprising that the overlapping APG/AUT/CVT genes are primarily involved in double-membrane vesicle formation (Stromhaug and Klionsky, 2001). Recent studies, however, have uncovered several genes specific for only one of the two pathways. Characterizing the different aspects of the two pathways will help us assign molecular functions to those newly cloned $A P G$ and $C V T$ genes.

Besides the aforementioned opposite metabolic natures, the most obvious difference between the Cvt and autophagy pathways that has been detected through morphological studies is the size of the sequestering vesicles. Cvt vesicles have an average diameter of $\sim 150 \mathrm{~nm}$, while autophagosomes range from 300-900 $\mathrm{nm}$ in diameter (Baba et al., 1997). It is not clear what components constitute the bound- 
ary membranes of these two vesicle types. If they share components, those molecules should be consumed at higher rates for autophagosome formation-autophagosomes are much more abundant under starvation conditions than Cvt vesicles are in rich media. A starvation-inducible property for an autophagy protein may very well indicate a structural role in vesicle formation.

Recent biochemical studies have unveiled subtle quantitative differences between the two pathways. An enzyme assay was developed to measure autophagy activity (Noda et al., 1995). PHO8 encodes the vacuolar membrane protein alkaline phosphatase. Deletion of its $\mathrm{N}$-terminal 60 residues eliminates the cytosolic domain and transmembrane segment of precursor Pho8 that acts as an internal uncleaved signal sequence allowing entry into the rER. The mutant form of the protein is unable to enter the rER and accumulates in the cytosol as an inactive enzyme. The only way to transport Pho8 860 to the vacuole is through doublemembrane vesicles formed in the cytoplasm. After entering the vacuole, hydrolytic processing activates the precursor enzyme. Measuring alkaline phosphatase activity allows an evaluation of the level of non-specific cytosolic protein transport to the vacuole. Under growth conditions, Pho8 $\Delta 60$ activity is kept at a minimal background level. After nutrient starvation to induce autophagy, Pho8 860 maturation reaches a plateau of $\sim 30 \%$ of total accumulated cytosolic Pho8 600 precursors after a three hour lag. These types of studies documented the inducible nature of the autophagy pathway. In contrast, Cvt pathway-mediated prApe1 import is a constitutive process. In addition, prApe 1 processing efficiency is kept at $\sim 100 \%$ through either pathway, suggesting some type of sorting or recognition mechanism for prApe1 delivery. Similarly, a sorting mechanism is likely to mediate efficient peroxisome turnover by pexophagy. Factors affecting delivery of specific cargo, such as prApe1 and peroxisomes, but not non-specific cytosolic substrates, such as Pho8 $\Delta 60$, are candidates of the sorting machinery for the Cvt and autophagy pathways.

Another difference between the Cvt and autophagy pathways is seen in the requirements of secretory and endocytic components. Autophagy, but not the Cvt pathway, requires the functions of the Sec12, Sec16, Sec23, and Sec24 proteins, which primarily participate in ER to Golgi transport (Ishihara et al., 2001). On the other hand, only the Cvt pathway is affected in $\operatorname{tg} 2$ and vps 45 mutants, strains deficient in the endosomal system (Abeliovich et al., 1999). These different requirements have been proposed to reflect the use of different membrane sources for the two pathways.

With prApe1 as a convenient marker for cytoplasm to vacuole transport studies, our knowledge about the Cvt and autophagy pathways has increased dramatically. The following sections will summarize the properties and proposed functions of the known autophagy and Cvt proteins.

\section{The machinery of the Cvt and autophagy pathways}

The Cvt pathway and autophagy operate under distinct physiological conditions. The starvation-inducible nature of autophagy suggests that an upstream nutrient sensor regulates the execution of this pathway. Downstream of the signaling complexes, general vesicle formation machinery cooperates with a cargo sorting system to regulate vesicle assembly and cargo loading. Completely sealed vesicles are then targeted to and fuse with the vacuole, a step that is regulated by general membrane trafficking machinery. It is critical that only completed vesicles fuse with the vacuole to ensure delivery of the cargo into the lumen. The final step of import requires vacuolar enzymes to lyse the subvacuolar vesicles, process prApe1, and in the case of autophagy to digest cargo and release building blocks into the cytosol for reuse in biosynthetic processes (Stromhaug and Klionsky, 2001).

\section{Signaling complexes}

Tor signaling mechanism-Tor (Target of rapamycin) is a phosphatidylinositol (PtdIns) kinase-related serine/threonine protein kinase that coordinates different aspects of cell physiology in response to nutrient conditions (Raught et al., 2001). Treatment of cells with the immunosupressant rapamycin not only causes cell cycle arrest at $\mathrm{G}_{0}$ (Rohde et al., 2001), but also induces autophagy (Noda and Ohsumi, 1998), suggesting Tor is an upstream nutrient sensor for the regulation of autophagy. Tor phosphorylates Tap42 in yeast, causing its association with protein phosphatase 2A (PP2A) (Beck and Hall, 1999). Tap42-associated PP2A has low enzymatic activity. Low nutrient stress or rapamycin treatment inhibits Tor, resulting in dephosphorylated Tap42 dissociating from PP2A. PP2A may then activate downstream growth arrest effectors, such as the transcription factor Gln3, and induce autophagy. In mammalian cells, PP2A activity correlates strongly with autophagy induction (Holen et al., 1992), supporting the involvement of this signaling pathway in autophagic regulation. Tap42 and Gln3, however, are not required for Tor-dependent regulation of autophagy in yeast, which suggests that a novel Tor signaling branch separates its general cell growth control from its role in autophagy (Kamada et al., 2000). This novel Tor signaling pathway may well involve Apg1 protein complexes, although the detailed signal transduction event is still not understood.

Apg1 and Apg13 complex-Apg1 and Apg13 are phosphoproteins required for both the Cvt pathway and autophagy (Kamada et al., 2000; Scott et al., 2000). Apg1 is a serine/threonine protein kinase (Matsuura et al., 1997; Straub et al., 1997), whose activity is stimulated by association with Apg13 (Kamada et al., 2000). Under nutrient rich conditions, active Tor causes hyperphosphorylation of 
Apg13, preventing or moderating its association with Apg1. It is not clear whether Tor directly phosphorylates Apg13. In nutrient starvation conditions or after treatment with rapamycin, Apg13 is quickly partially dephosphorylated, and its affinity for Apg1 dramatically increases in these situations. Based on in vitro studies, association of Apg1 with dephosphorylated Apg13 stimulates Apg1 kinase activity, which was proposed to signal a switch in the general vesicle formation machinery from the Cvt pathway to autophagy (Kamada et al., 2000). However, recent studies using chemical genetics to allow an in vivo assessment of the role of Apg1 kinase activity suggest that high kinase activity is required for the Cvt pathway but not for induction of autophagy (Abeliovich et al., 2003). Rather, Apg1 may have a structural role in autophagic induction. Many questions remain to be answered with regard to the conversion between these two pathways. For example, it is not clear how inactivated Tor regulates dephosphorylation of Apg13, but PP2A is not required. Neither is it known how Apg1 and Apg13 signal transduction controls the pathway that is utilized. Apg1 carries out autophosphorylation (Matsuura et al., 1997). An additional target for the Apg1 kinase has not yet been identified, but pathway-specific components, which associate with the Apg1 and Apg13 complexes, are believed to be crucial for the pathway selection processes.

Apgl and Apg13 complex-associated specific components-Vac8 and Cvt9 are phosphoproteins required for the import of prApe1 through the Cvt pathway (Kim et al., 2001b; Scott et al., 2000). In starvation conditions, the requirement of Cvt9 for prApe1 delivery by autophagosomes is less critical. About half of the accumulated prApe 1 is processed to its mature form upon switching $c v t 9 \Delta$ cells to nutrient-limited media. On the other hand, this strain carries out normal autophagy relative to a wild type strain based on the Pho8 860 enzymatic assay. These results indicate that Cvt9 is important for specific cargo delivery. This view is supported by the fact that Cvt9, or its homologue Gsa9, is also required for peroxisome degradation in both $S$. cerevisiae and P. pastoris (Kim et al., 2001b). How Cvt9 regulates specific cargo delivery is not clear, but it likely involves its interaction with Apg1. Unlike the $c v t 9$ mutant, autophagy activity is substantially diminished in vac $8 \Delta$ cells, yet prApe1 import is completely restored in a starved vac $8 \Delta$ strain (Scott et al., 2000). Vac8 interacts with Apg13. Further study is required to evaluate the importance of the interaction between these two proteins for prApe1 import. In addition to its function in prApe 1 import and the autophagy pathway, Vac8 is required for vacuole inheritance (Pan and Goldfarb, 1998; Wang et al., 1998). Thus, it is possible that Vac8 has a general role in vacuolar transport. This might account for the more severe autophagic defect relative to the ability to transport prApe1 during starvation.

While the Apg1-Apg13 complex interacts with components that are specific for the Cvt pathway, these proteins also interact with at least one component specific for autophagy. Apg17 is required for Apg13-dependent in vitro stimulation of Apg1 kinase activity, either by participating in or stabilizing the formation of the Apg1-Apg13 complex (Kamada et al., 2000). Despite the low Apg1 kinase activity in apg $17 \Delta$ cells, prApe1 import is only minimally affected in either growth or starvation conditions (Nice et al., 2002). The precise role of Apg17 in the regulation of autophagy remains to be determined, but the fact that overexpression of Apg1 rescues the autophagy defect in apg $17 \Delta$ cells supports the proposal that Apg1 acts downstream of Apg17. Together, these data suggest that Apg1 and Apg13 make a core complex for regulating the switch between the two pathways by associating with pathway-specific components. The model, however, must be an over-simplified view because Apg17 was recently found to interact with two new Cvt pathway-specific components, Cvt13 and Cvt20 (Nice et al., 2002; Uetz et al., 2000). It remains a challenge to evaluate the meaning of interactions between the autophagy-specific Apg17 and Cvt pathway-specific Cvt13 and Cvt20 proteins.

Phosphatidylinositol 3-phosphate signaling-Cvt13 and Cvt20 are both required for prApe1 import through the Cvt pathway (Nice et al., 2002). Under starvation conditions, only mild kinetic delays for prApe 1 processing were seen and no significant autophagy defects could be detected for either mutant strain. Cvt13 and Cvt20 both contain PX domains that bind phosphatidylinositol 3-phosphate (PtdIns(3)P) (Nice et al., 2002; Yu and Lemmon, 2001). Binding to PtdIns(3)P is important for the correct subcellular localization of the Cvt13 and Cvt20 proteins and in their functions in prApe1 delivery, indicating that Cvt13 and Cvt20 are downstream effectors of an autophagy-specific PtdIns 3-kinase signaling system. Cvt13 and Cvt20 both interact with Apg17 and thus may interact indirectly with the Apg1-Apg13 complex. This set of interactions brings the two autophagy signaling systems, PtdIns 3-kinase and Apg1 kinase, together for the first time.

The budding yeast $S$. cerevisiae has only one PtdIns 3kinase, Vps34. Other than regulation of autophagy and the Cvt pathway, the majority of the PtdIns(3)P signal generated by Vps34 is involved in conventional vacuolar protein targeting through the endosome/prevacuolar compartment (Stack et al., 1993; Stack and Emr, 1994). Vps34 participates in regulating those pathways by interaction with specific components. Vps15 is a membrane associated protein kinase that forms a core complex with Vps34 and regulates its activity (Stack et al., 1995; Stack et al., 1993). For its conventional vacuolar protein sorting function, the Vps15Vps34 complex further interacts with two additional components, Vps30 and Vps38. The exact compartment where this Vps pathway-specific PtdIns 3-kinase complex acts is not clear, but it is proposed to regulate recycling of the Prc1 receptor, Vps10, from the endosome/prevacuolar compartment back to the late-Golgi. To regulate autophagy, Vps 15 and Vps34 interact with another protein complex, Vps30 
and Apg14 (Kametaka et al., 1998; Kihara et al., 2001). Apg14, and by extension the autophagy-specific PtdIns 3kinase complex, localizes at a pre-autophagosomal structure (PAS) close to the vacuole (Kim et al., 2002; Suzuki et al., 2001). The Cvt13 and Cvt20 proteins no longer localize at

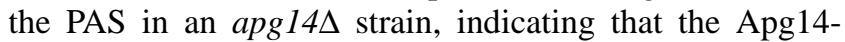
containing PtdIns 3-kinase complex carries out a function at this location (Nice et al., 2002). It is reasonable to speculate that pathway-specific components confer localization specificities for the Vps34 complexes. The Vps34 kinase product, PtdIns(3)P then recruits specific effectors to corresponding compartments for further action. It therefore is important to study how effectors distinguish between the different compartments that contain PtdIns(3)P. Both Cvt13 and Cvt20 have relatively weak affinities toward PtdIns(3)P and other factors may be required for their recruitment (Nice et al., 2002).

Another PtdIns(3)P binding protein, Etf1, required for prApe 1 import by the Cvt pathway was isolated from a screen for enhancers of the temperature conditional defect of a vps34ts strain (Wurmser and Emr, 2002). Despite the initial goal to identify Vps34 downstream effectors involved in conventional vacuolar protein transport, the only characterized phenotype of the etfl $\Delta$ strain is a block of prApe 1 processing under growing conditions. Autophagy progresses normally in the etfl $\Delta$ strain, and prApe 1 import is restored after starvation. Etf1 encodes a type II transmembrane protein without a known PtdIns(3)P binding motif, such as the FYVE or PX domains. A basic residue patch proximal to the transmembrane anchor, however, is both involved in its PtdIns(3)P binding activity and Cvt pathway function. Etf1 co-immunoprecipitates with Aut7, a Cvt and autophagic vesicle component, indicating a potential link between a signaling event and sequestering vesicle formation. The isolation of Etf1 provides an exciting direction to dissect PtdIns(3)P signaling in the regulation of the Cvt pathway, and much work remains to integrate Etf1 into the current regulatory schemes.

\section{General vesicle formation machinery}

Unlike signaling complexes, which usually associate with specific components to regulate switching between the $\mathrm{Cvt}$ and autophagy pathways, the vesicle formation machinery has a more general role in the assembly of the doublemembrane transport vesicle. Mutants in this category affect both the Cvt and autophagy pathways and block vesicle formation at a stage before its completion. Among the characterized components, proteins in this group can be further separated into functional subgroups, including two novel conjugation systems and a putative integral membrane protein complex.

Apg12-Apg5 conjugation system-Apg5, Apg7, Apg10, Apg12, and Apg16 constitute an ubiquitin-like conjugation system (Ohsumi, 2001). The Apg12 C-terminal carboxyl group is covalently linked to Apg5 through the amide group of the Lys ${ }^{149}$ side chain (Mizushima et al., 1998a). The formation of this isopeptide bond is catalyzed sequentially by Apg7 and Apg10 (Shintani et al., 1999; Tanida et al., 1999). Apg7 first activates Apg12 by forming a thioester bond between the Apg12 C-terminal carboxyl group and the $\mathrm{Cys}^{507}$ side chain using energy provided by ATP hydrolysis. Apg7 is therefore equivalent to the E1 enzyme of the ubiquitination system. Apg12 is subsequently transferred to Apg10 from Apg7, and a similar thioester bond is formed with $\mathrm{Cys}^{133}$ of Apg10. Like the ubiquitin system, Apg12 is finally conjugated to Apg5 through the formation of a stable isopeptide bond. Despite its parallel mechanism to the ubiquitin system, there are some distinct aspects for this autophagy conjugation machinery. First of all, no E3 enzyme has been identified for this system so far and it is likely that Apg10 directly catalyzes the final step of isopeptide bond formation. Apg12 does not show any significant sequence similarities to ubiquitin or its related small protein modifiers. Apg7 has only limited similarity to other E1 enzymes, and it is mostly confined at the catalytic domain. Finally, the only target of Apg12 identified so far is Apg5. Immunoblot analyses indicate that the Apg12-Apg5 conjugate is the major, if not the only modification product.

Another autophagy protein involved in this conjugation system is Apg16 (Mizushima et al., 1999). Apg16 is a small protein with a predicted coiled-coil domain required for its homo-oligomerization and autophagy function. In addition, Apg16 interacts with Apg5 resulting in a multi-protein complex. The majority of Apg12 protein is covalently linked to Apg5 at steady state, and Apg16 oligomerization causes the three components to exist as a $\sim 350 \mathrm{kDa}$ complex (Kuma et al., 2002). The complex is proposed to be composed of Apg5, Apg12 and Apg16 in a tetrameric stoichiometry. It is an enigma as to how this protein conglomerate regulates double-membrane vesicle formation, and to answer this question requires more detailed information about the dynamics of the complex assembly.

The mammalian homologue of the Apg5 protein (hApg5) is also covalently modified by the Apg12 homologue (hApg12) (Mizushima et al., 1998b), and has been found to localize to cytosolic forming autophagosomes (Mizushima et al., 2001). Before or right after the completion of vesicle formation, mouse Apg5 is quickly released from the autophagosomes. The mechanism of this quick mouse Apg5 release is not known, but it probably does not require separation of covalently bound Apg5 and Apg12, because a factor with Apg12 C-terminal processing activity could not be identified. Unlike the ubiquitin system, where deubiquitination enzymes also process nascent polyubiquitin translation products, Apg12 is synthesized as a monomer and fusions at its $\mathrm{C}$ terminus block its function, suggesting the lack of an Apg12 de-conjugation mechanism. The actual physiological function of the Apg12 conjugation system is not known, but recent studies indicate that it is required for 
the formation of a novel Aut7-lipid conjugate (Kim et al., 2001a; Ichimura et al., 2000).

Aut7 conjugation system-Aut7 is the first characterized starvation-inducible autophagy protein (Huang et al., 2000;

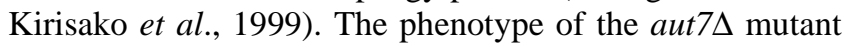
reveals its involvement in vesicle formation in the Cvt pathway. In contrast, Aut7 does not appear to play an essential role in vesicle formation during autophagy. Precursor Ape1 is partially matured under starvation conditions and aut7D cells display an intermediate phenotype compared to apg $1 \Delta$ cells with regard to starvation-sensitivity, an assay for autophagy activity (Abeliovich et al., 2000). Inhibition of Aut7 induction with cycloheximide does not prevent the formation of autophagosomes. However, the resulting autophagosomes are abnormally small, suggesting that Aut7 induction is needed for expansion of the autophagosome membrane (Abeliovich et al., 2000). Aut7 is delivered together with the double-membrane transport vesicles to the vacuole and turned over by vacuolar proteases. Together, these data suggest a structural function for Aut7 in vesicle formation but indicate differences between the formation of Cvt vesicles and autophagosomes. Further studies are required to understand how Aut7 regulates vesicle formation in both pathways.

In living cells, a functional GFP-Aut7 chimera is localized to the PAS in addition to completely formed autophagosomes and Cvt vesicles (Kim et al., 2001a). The PAS is believed to be a physiological intermediate structure for prApe1 import and vesicle formation. GFP-Aut7 localization to the PAS is blocked in mutants deficient in the Apg12 conjugation system. This is the first direct defect linked to the Apg 12 conjugation mutant strains. A detailed interaction map will certainly help us to refine the role of the Apg12 conjugation system in Aut7 recruitment to the forming vesicles.

Another group of autophagy mutants affecting Aut7 recruitment to the PAS includes the autl and aut2 strains (Kim et al., 2001a; Kirisako et al., 1999). Ohsumi and colleagues discovered that these proteins constituted another novel autophagy conjugation system (Ichimura et al., 2000). Aut7 is synthesized as a soluble precursor containing a Cterminal arginine. This arginine must be removed by Aut2, a cysteine proteinase, before $\mathrm{Apg} 7$ can activate Aut7 in a manner similar to Apg12 activation. The newly revealed Aut7 glycine residue is critical for the subsequent conjugation reaction, probably having a role in enzymatic recognition. The new Aut7 C-terminal carboxyl group is linked to Apg7 $\mathrm{Cys}^{507}$, and then transferred to Aut1 $\mathrm{Cys}^{234}$, the E2 enzyme of this system. The final target of the Aut7 modifier is the lipid molecule phosphatidylethanolamine (PE). PE is an abundant lipid asymmetrically distributed in the inner leaflet of unit membranes. After transport vesicle formation is complete, inner membrane surface-bound Aut7 is trapped inside of the vesicles and successively transported to and degraded in the vacuole. The outer membrane associated
Aut7 has to be removed by Aut2 for efficient vesicle processing. This Aut7 modifier is a novel system using an ubiquitin-like mechanism and yet having a lipid as a final target molecule. Further studies on the Aut7 system will certainly provide us with new insights in biological membrane trafficking.

Aut7 has three mammalian homologues, microtubuleassociated protein light chain 3 (MAP-LC3), the Golgi associated ATPase enhancer of $16 \mathrm{kDa}$ (GATE-16), and $\gamma$ aminobutyric acid receptor-associated protein (GABARAP). GATE-16 can enhance intra-Golgi protein transport in vitro (Legesse-Miller et al., 2000). GABARAP associates with GABA receptor and cytoskeleton, and is proposed to participate in receptor endocytosis (Wang et al., 1999). MAPLC3 was originally isolated as a microtubule-associated protein (Mann and Hammarback, 1994), and was recently found localized to and required for the formation of mammalian autophagosomes (Kabeya et al., 2000). The three mammalian Aut7 homologues are all modified by mammalian Apg7 and Aut1 (Tanida et al., 2002; Tanida et al., 2001), suggesting that they are derived from the same conserved system as the yeast Aut7 modifier. The target molecules of the three mammalian modifier systems remain to be determined.

A putative Apg9 membrane protein complex-Apg9 is a multi-spanning transmembrane protein (Noda et al., 2000). The function of Apg9 is required for both autophagosome and Cvt vesicle formation, but unlike Aut7, Apg9 is excluded from completed transport vesicles. GFP fusion and immunofluorescent signals locate Apg9 at the PAS together with several other autophagy proteins, including Aut7 (Kim et al., 2002; Suzuki et al., 2001). This localization plus its nature as an integral membrane protein make Apg9 an ideal marker of the membrane source for making Cvt and autophagic vesicles. Apg9 is found to interact with Apg2, based on immunoaffinity isolation using Apg9 tagged at its $\mathrm{N}$ terminus with an IgG binding motif of protein A (Wang et al., 2001). An apg2 mutant shows a defect in vesicle formation (Shintani et al., 2001; Wang et al., 2001). The localization of Apg 2 to the PAS requires both Apg9 and Apg1, although Apg1 kinase activity does not seem to be critical for this function. Recently, Cvt18 was cloned and found to be required for vesicle formation (Guan et al., 2001). Cvt18 localizes to the PAS and vacuolar rim independent of other autophagy proteins. However, in a cvt18 18 mutant, Apg2 localization is impaired, suggesting a potential functional link with the Apg9 system. Taken together, the data suggest that Apg9 may provide different autophagy protein complexes with a docking site for binding to the PAS, and possibly integrate their functions for vesicle formation.

Cargo loading and vesicle formation-Cargo loading is an important triggering signal for transport vesicle formation in endomembrane and plasma membrane systems (Le Borgne et al., 1996; Le Borgne and Hoflack, 1997). For the 
Cvt and autophagy pathways, the prApe1-containing Cvt complex is a cargo being efficiently sorted into the doublemembrane transport vesicles. This process requires a newly characterized protein, Cvt19, which is induced similar to prApe1 by starvation stimuli and turned over in the vacuole (Scott et al., 2001). Cvt19 specifically interacts with the propeptide of prApe1 and Ams1, and this interaction tightens the membrane-associated status of prApe1. In the absence of Cvt19, prApe1 and Ams1 are not efficiently delivered to the vacuole under nutrient-rich or starvation conditions despite the fact that autophagy operates normally in the $c v t 19 \Delta$ strain judging by non-specific transport of Pho8 $\Delta 60$. Interestingly, Cvt19 turnover by the vacuole is slowed down in the ape $1 \Delta$ strain, which indicates that the association of Cvt19 and prApe1 promotes the vacuolar delivery of both proteins. The Cvt complex is not the only cargo of autophagosomes, so it is not surprising that the absence of Cvt19 and/or prApe1 does not affect the autophagy pathway. It remains to be determined whether $\mathrm{Cvt}$ complex loading triggers the formation of $\mathrm{Cvt}$ vesicles.

\section{Docking and fusion machinery}

Completely formed Cvt vesicles and autophagosomes must be targeted to the vacuole for fusion. In the past decade, several conserved protein families have been found to be responsible for membrane fusion specificity and efficiency. In the case of yeast vacuole fusion, the docking and fusion machinery includes: 1) SNARE proteins: Vam3, Vam7, Vti1, Ykt6 and Nyv1; 2) a Rab family GTPase: Ypt7; and 3) the class $\mathrm{C}$ Vps protein complex, also known as the HOPS (homotypic fusion and vacuole protein sorting) complex (Wickner and Haas, 2000). The involvement of these proteins in Cvt vesicle and autophagosome fusion with the vacuole has been tested. Not surprisingly, most examined strains showed defects in prApe1 processing and autophagy activity (Fischer von Mollard and Stevens, 1999; Harding et al., 1995; Nakamura et al., 1997a; Sato et al., 1998). Further evidence is provided by studies with Ccz1. Ccz1 is an Ypt7 interacting protein required for homotypic vacuolar fusion and conventional vacuolar hydrolase transport (Kucharczyk et al., 2000; Kucharczyk et al., 2001). Overexpression of Ypt7 in a $c c z 1 \Delta$ strain suppresses the fragmented vacuole phenotype and restores vacuolar hydrolase import. In a $c c z 1$ mutant, prApe 1 import is blocked at a stage after completion of vesicle formation indicating a defect in vacuolar fusion (Meiling-Wesse et al., 2002; Wang et al., 2002). These results, however, should be considered with caution because the vacuolar fusion mutant strains also have defects in general vacuolar enzyme processing, and the blocks in the Cvt and autophagy pathways could be indirect. Nevertheless, the accumulation of cytosolic autophagosomes and Cvt vesicles in a vam $3^{\text {ts }}$ strain at non-permissive temperature validates the direct involvement of vacuolar fusion machinery in the Cvt and autophagy pathways (Abeliovich et al., 2000). This conclusion raises a question as to how the fusion components, such as SNARE proteins, are recruited to $\mathrm{Cvt}$ vesicles and autophagosomes. Identifying the membrane sources for vesicle formation is the key to answering this question.

\section{Vacuolar processing machinery}

Inside the vacuolar lumen, before proteases can access their substrates, a presumptive lipase activity is required to degrade the inner membrane boundary of Cvt and autophagic bodies. The cvt17 mutant accumulates subvacuolar vesicles, including $\mathrm{Cvt}$ and autophagic bodies as well as vesicles derived from the MVB pathway (Epple et al., 2001; Teter et al., 2001). Cvt17 is an integral membrane protein with a conserved lipase domain. A conserved active site amino acid is essential for its function in subvacuolar vesicle degradation, confirming its role as a lipase. However, it is not known if Cvt17 function is required within the vacuole or at the site of vesicle formation. In class $\mathrm{E} v p s$ mutants, Cvt17 is accumulated in the prevacuolar compartment (PVC), which suggests that Cvt17 is delivered to the vacuolar lumen through MVB vesicles. The fact that the import of this protein into the vacuole lumen is also blocked in a fabld mutant suggests that Cvt17 is ubiquitinated or associates with an ubiquitinated protein in order to enter MVB vesicles (Reggiori and Pelham, 2002). Cvt17 has a single transmembrane segment and the bulk of the protein is lumenal. Its short cytosolic tail contains 16 amino acids with two lysine residues that could act as ubiquitination sites. This structure is very similar to that of Cps1 and Phm5, two proteins that use the MVB pathway to reach the vacuole interior (Katzman et al., 2001; Reggiori and Pelham, 2001), further supporting the idea that Cvt17 is a cargo of MVB vesicles.

Another recently cloned gene, AUT4, has also been shown to play a role in subvacuolar vesicle degradation (Suriapranata et al., 2000). Aut4 is a multipass transmembrane protein with the majority of its residues embedded in the membrane. The function of this protein is still not clear, but it is likely to be distinct from that of Cvt17. An aut4s mutant only affects autophagic body degradation, and yet Aut4 localizes to perivacuolar punctate structures and the vacuolar membrane, seemingly excluding a role in acting directly on autophagic bodies. It is a puzzle as to how Aut4 localized to the vacuolar membrane affects the degradation of only one type of subvacuolar vesicle. Maybe the perivacuolar punctate Aut4 structures correspond to the membrane source for autophagosome formation. Constraining Aut 4 to the proposed autophagosome-specific membrane source would account for the different Aut 4 effects for the two pathways. Aut 4 incorporated into the autophagosome may be present at too low a level to detect by fluorescent microscopy.

The final stage of substrate degradation and prApe 1 pro- 
cessing requires protease activities. The vacuolar proteinase A (Pep4) is the master protease in the system (Klionsky et al., 1990). Upon reaching the vacuole, the acidic environment causes autoactivation of Pep4. Pep4 subsequently activates other vacuolar proteases, resulting in a cascade that equips the vacuole with a full complement of active enzymes. The low $\mathrm{pH}$ environment controlled by the vacuolar ATPase is important for establishing and maintaining vacuolar hydrolytic ability, and for the efficient degradation of Cvt and autophagic bodies (Nakamura et al., 1997b).

\section{Conclusion and future directions}

Our knowledge of autophagy in yeast has increased dramatically in the past decade. One important landmark in this progress was the discovery that the import machinery for the vacuolar enzyme Ape1, the Cvt components, partially overlaps with that of autophagy (Harding et al., 1996; Scott et al., 1996). Using prApe1 processing as an easily followed marker, the molecular functions of autophagy and Cvt components can be organized into several functional groups. In the near future, one major challenge is to understand how the different functional groups communicate with each other and coordinate the events for successfully transporting cytosolic cargo to the vacuole.

At least Tor protein- and phosphoinositide lipid-mediated signaling events are known to regulate the switch between the Cvt pathway and autophagy (Nice et al., 2002; Kihara et al., 2001; Kamada et al., 2000; Noda and Ohsumi, 1998). Several autophagy and Cvt proteins are involved in signal transduction, but our current knowledge can only assemble them into two largely separated systems (Stromhaug and Klionsky, 2001). What are the downstream effectors of the Tor protein-regulated Apg1-Apg13 complexes? Is the Apg14-associated phosphoinositide system downstream or upstream of the Apg1-Apg13 system? How do they cross talk with each other? How do they coordinate the general vesicle formation machinery? The answers to some of these questions may come from future studies on recently identified phosphoinositide binding proteins, Cvt13 and Cvt20, which also interact with components of the Apg1-Apg13 signaling system (Nice et al., 2002).

General vesicle formation machinery for the Cvt pathway and autophagy can be placed into three functional groups: 1) Apg9 and associated proteins constitute a putative membrane protein complex (Wang et al., 2001). What is the identity of the Apg9 localized membrane structure? How is Apg9 transported to the final membrane domain? How is Apg9 excluded from completely formed double-membrane transport vesicles? 2) Apg12 conjugation machinery (Mizushima et al., 1998a). How is the Apg12 system recruited to the vesicle formation site? What is the identity of the membrane source for vesicle formation? How does the Apg12 conjugation machinery regulate membrane recruitment of Aut7? What stage of Aut7-PE conjugate formation is regulated by the Apg12 system? 3) Aut7 is covalently bound to PE (Ichimura et al., 2000). How does Aut7 lipidation affect vesicle formation? Studies on the Aut7 mammalian homologue GATE-16 may shed light on this question. GATE-16 is found to interact with the Golgi SNARE protein GOS28 and is proposed to regulate intraGolgi transport (Muller et al., 2002). Interestingly, Aut7 also interacts with yeast Golgi and vacuolar SNARE proteins, such as Bet1 and Nyv1 (Legesse-Miller et al., 2000). It is an unexplored issue whether these interactions mediate Cvt and autophagy vesicle formation.

Targeting and fusion of completely formed Cvt vesicles and autophagosomes to the vacuole requires the general vacuolar fusion machinery. How are v-SNAREs recruited to the transport vesicles? Do these fusion proteins recycle? What is the membrane trafficking route for this recycling pathway? Inside the vacuole, the membrane boundary of Cvt and autophagic bodies is degraded by vacuolar hydrolases. This process requires the function of Cvt17 (Teter et al., 2001). How might Cvt17 modify the inner membrane of transport vesicles leaving the vacuolar limiting membrane unaffected? How is breakdown of the outer vesicle membrane regulated to preserve vacuolar integrity? All these questions remained to be answered and, undoubtedly, solving theses issues will continue to bring us new and exciting questions for the future.

Acknowledgments. The authors thank Dr. Fulvio Reggiori for helpful comments on the manuscript. This work was supported by National Institutes of Health Public Health Service grant GM53396 to D.J. Klionsky.

\section{References}

Abeliovich, H., Darsow, T., and Emr, S.D. 1999. Cytoplasm to vacuole trafficking of aminopeptidase I requires a t-SNARE-Sec1p complex composed of Tlg2p and Vps45p. EMBO J., 18: 6005-6016.

Abeliovich, H, Dunn, W.A., Jr., Kim, J., and Klionsky, D.J. 2000. Dissection of autophagosome biogenesis into distinct nucleation and expansion steps. J. Cell Biol., 151: 1025-1034.

Abeliovich, H. and Klionsky, D.J. 2001. Autophagy in yeast: mechanistic insights and physiological function. Microbiol. Mol. Biol. Rev., 65: 463479.

Abeliovich, H., Zhang, C., Dunn, W.A., Jr., Shokat, K.M., and Klionsky, D.J. 2003. Chemical genetic analysis of Apg1 reveals a non-kinase role in the induction of autophagy. Mol. Biol. Cell, in press.

Agarraberes, F.A. and Dice, J.F. 2001. A molecular chaperone complex at the lysosomal membrane is required for protein translocation. J. Cell Sci., 114: 2491-2499.

Baba, M., Osumi, M., Scott, S.V., Klionsky, D.J., and Ohsumi, Y. 1997. Two distinct pathways for targeting proteins from the cytoplasm to the vacuole/lysosome. J. Cell Biol., 139: 1687-1695.

Beck, T. and Hall, M.N. 1999. The TOR signalling pathway controls nuclear localization of nutrient-regulated transcription factors. Nature, 402: 689-692.

Brown, C.R., McCann, J.A., and Chiang, H.-L. 2000. The heat shock protein $\mathrm{Ssa} 2 \mathrm{p}$ is required for import of fructose-1,6-bisphosphatase into Vid vesicles. J. Cell Biol., 150: 65-75.

Bryant, N.J., and Stevens, T.H. 1998. Vacuole biogenesis in Saccharomyces cerevisiae: protein transport pathways to the yeast vacuole. 
Microbiol. Mol. Biol. Rev., 62: 230-247.

Dice, J.F. 1992. Selective degradation of cytosolic proteins by lysosomes. Ann. N.Y. Acad. Sci., 674: 58-64.

Epple, U.D., Suriapranata, I., Eskelinen, E.L., and Thumm, M. 2001. Aut5/Cvt17p, a putative lipase essential for disintegration of autophagic bodies inside the vacuole. J. Bacteriol., 183: 5942-5955.

Fischer von Mollard, G., and Stevens, T.H. 1999. The Saccharomyces cerevisiae $\mathrm{v}$-SNARE Vti1p is required for multiple membrane transport pathways to the vacuole. Mol. Biol. Cell, 10: 1719-1732.

Guan, J., Stromhaug, P.E., George, M.D., Habibzadegah-Tari, P., Bevan, A., Dunn, W.A., Jr., and Klionsky, D.J. 2001. Cvt18/Gsa12 is required for cytoplasm-to-vacuole transport, pexophagy, and autophagy in Saccharomyces cerevisiae and Pichia pastoris. Mol. Biol Cell, 12: 3821-3838.

Harding, T.M., Hefner-Gravink, A., Thumm, M., and Klionsky, D.J. 1996. Genetic and phenotypic overlap between autophagy and the cytoplasm to vacuole protein targeting pathway. J. Biol. Chem., 271: 17621-17624.

Harding, T.M., Morano, K.A., Scott, S.V., and Klionsky, D.J. 1995. Isolation and characterization of yeast mutants in the cytoplasm to vacuole protein targeting pathway. J. Cell Biol., 131: 591-602.

Holen, I., Gordon, P.B., and Seglen, P.O. 1992. Protein kinase-dependent effects of okadaic acid on hepatocytic autophagy and cytoskeletal integrity. Biochem. J., 284: 633-636.

Horst, M, Knecht, E.C., and Schu, P.V. 1999. Import into and degradation of cytosolic proteins by isolated yeast vacuoles. Mol. Biol. Cell, 10: 2879-2889

Huang, P.H. and Chiang, H.-L. 1997. Identification of novel vesicles in the cytosol to vacuole protein degradation pathway. J. Cell Biol., 136: 803810.

Huang, W.-P., Scott, S.V., Kim, J., and Klionsky, D.J. 2000. The itinerary of a vesicle component, Aut $7 \mathrm{p} / \mathrm{Cvt} 5 \mathrm{p}$, terminates in the yeast vacuole via the autophagy/Cvt pathways. J. Biol. Chem., 275: 5845-5851.

Ichimura, Y., Kirisako, T., Takao, T., Satomi, Y., Shimonishi, Y., Ishihara, N., Mizushima, N., Tanida, I., Kominami, E., Ohsumi, M., Noda, T., and Ohsumi, Y. 2000. A ubiquitin-like system mediates protein lipidation. Nature, 408: 488-492.

Ishihara, N., Hamasaki, M., Yokota, S., Suzuki, K., Kamada, Y., Kihara, A., Yoshimori, T., Noda, T., and Ohsumi, Y. 2001. Autophagosome requires specific early Sec proteins for its formation and NSF/SNARE for vacuolar fusion. Mol. Biol. Cell, 12: 3690-3702.

Kabeya, Y., Mizushima, N., Ueno, T., Yamamoto, A., Kirisako, T., Noda, T., Kominami, E., Ohsumi, Y., and Yoshimori, T. 2000. LC3, a mammalian homologue of yeast Apg8p, is localized in autophagosome membranes after processing. EMBO J., 19: 5720-5728.

Kamada, Y., Funakoshi, T., Shintani, T., Nagano, K., Ohsumi, M., and Ohsumi, Y. 2000. Tor-mediated induction of autophagy via an Apg1 protein kinase complex. J. Cell Biol., 150: 1507-1513.

Katzman, D.J., Babst, M., and Emr, S.D. 2001. Ubiquitin-dependent sorting into the multivesicular body pathway requires the function of a conserved endosomal protein sorting complex, ESCRT-I. Cell, 106: $145-155$

Kihara, A., Noda, T., Ishihara, N., and Ohsumi, Y. 2001. Two distinct Vps34 phosphatidylinositol 3-kinase complexes function in autophagy and carboxypeptidase Y sorting in Saccharomyces cerevisiae. J. Cell Biol., 152: 519-530.

Kim, J. Huang, W.-P., and Klionsky, D.J. 2001a. Membrane recruitment of Aut $7 \mathrm{p}$ in the autophagy and cytoplasm to vacuole targeting pathways requires Aut1p, Aut2p, and the autophagy conjugation complex. J. Cell Biol., 152: 51-64.

Kim, J., Kamada, Y., Stromhaug, P.E., Guan, J., Hefner-Gravink, A., Baba, M., Scott, S.V., Ohsumi, Y., Dunn, W.A., Jr., and Klionsky, D.J. 2001b. Cvt9/Gsa9 functions in sequestering selective cytosolic cargo destined for the vacuole. J. Cell Biol., 153: 381-396.
Kim, J., Huang, W.-P., Stromhaug, P.E., and Klionsky, D.J. 2002. Convergence of multiple autophagy and cytoplasm to vacuole targeting components to a perivacuolar membrane compartment prior to de novo vesicle formation. J. Biol. Chem., 277: 763-773.

Kim, J. and Klionsky, D.J. 2000. Autophagy, cytoplasm-to-vacuole targeting pathway, and pexophagy in yeast and mammalian cells. Аnпu. Rev. Biochem., 69: 303-342.

Kim, J., Scott, S.V., and Klionsky, D.J. 2000. Alternative protein sorting pathways. Int. Rev. Cytol., 198: 153-201.

Kim, J., Scott, S.V., Oda, M.N., and Klionsky, D.J. 1997. Transport of a large oligomeric protein by the cytoplasm to vacuole protein targeting pathway. J. Cell Biol., 137: 609-618.

Kirisako, T., Baba, M., Ishihara, N., Miyazawa, K., Ohsumi, M., Yoshimori, T., Noda, T., and Ohsumi, Y. 1999. Formation process of autophagosome is traced with Apg8/Aut7p in yeast. J. Cell Biol., 147 435-446.

Klionsky, D.J. Cueva, R., and Yaver, D.S. 1992. Aminopeptidase I of Saccharomyces cerevisiae is localized to the vacuole independent of the secretory pathway. J. Cell Biol., 119: 287-299.

Klionsky, D.J. and Emr, S.D. 2000. Autophagy as a regulated pathway of cellular degradation. Science, 290: 1717-1721.

Klionsky, D.J., Herman, P.K., and Emr, S.D. 1990. The fungal vacuole: composition, function, and biogenesis. Microbiol. Rev., 54: 266-292.

Kucharczyk, R., Dupre, S., Avaro, S., Haguenauer-Tsapis, R., Slonimski, P.P., and Rytka, J. 2000. The novel protein Ccz1p required for vacuolar assembly in Saccharomyces cerevisiae functions in the same transport pathway as Ypt7p. J. Cell Sci., 113: 4301-4311.

Kucharczyk, R., Kierzek, A.M., Slonimski, P.P., and Rytka, J. 2001. The Ccz1 protein interacts with Ypt7 GTPase during fusion of multiple transport intermediates with the vacuole in S. cerevisiae. J. Cell Sci., 114: 3137-3145.

Kuma, A., Mizushima, N., Ishihara, N., and Ohsumi, Y. 2002. Formation of the approximately 350-kDa Apg12-Apg5 Apg16 multimeric complex, mediated by Apg16 oligomerization, is essential for autophagy in yeast. J. Biol. Chem., 277: 18619-18625.

Le Borgne, R., Griffiths, G., and Hoflack, B. 1996. Mannose 6-phosphate receptors and ADP-ribosylation factors cooperate for high affinity interaction of the AP-1 Golgi assembly proteins with membranes. J. Biol. Chem., 271: 2162-2170.

Le Borgne, R. and Hoflack, B. 1997. Mannose 6-phosphate receptors regulate the formation of clathrin-coated vesicles in the TGN. J. Cell Biol., 137: 335-345.

Legesse-Miller, A., Sagiv, Y., Glozman, R., and Elazar, Z. 2000. Aut7p, a soluble autophagic factor, participates in multiple membrane trafficking processes. J. Biol. Chem., 275: 32966-32973.

Mann, S.S. and Hammarback, J.A. 1994. Molecular characterization of light chain 3. A microtubule binding subunit of MAP1A and MAP1B. J. Biol. Chem., 269: 11492-11497.

Matsuura, A., Tsukada, M., Wada, Y., and Ohsumi, Y. 1997. Apg1p, a novel protein kinase required for the autophagic process in Saccharomyces cerevisiae. Gene, 192: 245-250.

Meiling-Wesse, K., Barth, H., and Thumm, M. 2002. Ccz1p/Aut11p/ Cvt16p is essential for autophagy and the cvt pathway. FEBS Lett., 526: $71-76$.

Mizushima, N., Noda, T., and Ohsumi, Y. 1999. Apg16p is required for the function of the Apg12p-Apg5p conjugate in the yeast autophagy pathway. EMBO J., 18: 3888-3896.

Mizushima, N., Noda, T., Yoshimori, T., Tanaka, Y., Ishii, T., George, M.D., Klionsky, D.J., Ohsumi, M., and Ohsumi, Y. 1998a. A protein conjugation system essential for autophagy. Nature, 395: 395-398.

Mizushima, N., Sugita, H., Yoshimori, T., and Ohsumi, Y. 1998b. A new protein conjugation system in human. The counterpart of the yeast Apg12p conjugation system essential for autophagy. J. Biol. Chem., 273 
33889-33892.

Mizushima, N., Yamamoto, A., Hatano, M., Kobayashi, Y., Kabeya, Y., Suzuki, K., Tokuhisa, T., Ohsumi, Y., and Yoshimori, T. 2001. Dissection of autophagosome formation using Apg5-deficient mouse embryonic stem cells. J. Cell Biol., 152: 657-668.

Muller, J.M., Shorter, J., Newman, R., Deinhardt, K., Sagiv, Y., Elazar, Z., Warren, G., and Shima, D.T. 2002. Sequential SNARE disassembly and GATE-16-GOS-28 complex assembly mediated by distinct NSF activities drives Golgi membrane fusion. J. Cell Biol., 157: 1161-1173.

Nakamura, N., Hirata, A., Ohsumi, Y., and Wada, Y. 1997a. Vam2/ Vps41p and Vam6/Vps39p are components of a protein complex on the vacuolar membranes and involved in the vacuolar assembly in the yeast Saccharomyces cerevisiae. J. Biol. Chem., 272: 11344-11349.

Nakamura, N., Matsuura, A., Wada, Y., and Ohsumi, Y. 1997b. Acidification of vacuoles is required for autophagic degradation in the yeast, Saccharomyces cerevisiae. J. Biochem., 121: 338-344.

Nice, D.C., Sato, T.K., Stromhaug, P.E., Emr, S.D., and Klionsky, D.J. 2002. Cooperative binding of the cytoplasm to vacuole targeting pathway proteins, Cvt13 and Cvt20, to PtdIns(3)P at the pre-autophagosomal structure is required for selective autophagy. J. Biol. Chem., 277: 30198-30207.

Noda, T., Kim, J., Huang, W.-P., Baba, M., Tokunaga, C., Ohsumi, Y., and Klionsky, D.J. 2000. Apg9p/Cvt7p is an integral membrane protein required for transport vesicle formation in the Cvt and autophagy pathways. J. Cell Biol., 148: 465-480.

Noda, T. Matsuura, A., Wada, Y., and Ohsumi, Y. 1995. Novel system for monitoring autophagy in the yeast Saccharomyces cerevisiae. Biochem. Biophys. Res. Commun., 210: 126-132.

Noda, T. and Ohsumi, Y. 1998. Tor, a phosphatidylinositol kinase homologue, controls autophagy in yeast. J. Biol. Chem., 273: 3963-3966.

Noda, T., Suzuki, K., and Ohsumi, Y. 2002. Yeast autophagosomes: de novo formation of a membrane structure. Trends Cell Biol., 12: 231235.

Ohsumi, Y. 2001. Molecular dissection of autophagy: two ubiquitin-like systems. Nat. Rev. Mol. Cell. Biol., 2: 211-216.

Oliver, J., Jungnickel, B., Gorlich, D., Rapoport, T., and High, S. 1995. The Sec61 complex is essential for the insertion of proteins into the membrane of the endoplasmic reticulum. FEBS Lett., 362: 126-130.

Pan, X. and Goldfarb, D.S. 1998. YEB3/VAC8 encodes a myristylated armadillo protein of the Saccharomyces cerevisiae vacuolar membrane that functions in vacuole fusion and inheritance. J. Cell Sci., 111: 21372147.

Raught, B., Gingras, A.C., and Sonenberg, N. 2001. The target of rapamycin (TOR) proteins. Proc. Natl. Acad. Sci. USA, 98: 7037-7044.

Reggiori, F. and Pelham, H.R. 2002. A transmembrane ubiquitin ligase required to sort membrane proteins into multivesicular bodies. Nat. Cell. Biol., 4: 117-123.

Rohde, J., Heitman, J., and Cardenas, M.E. 2001. The TOR kinases link nutrient sensing to cell growth. J. Biol. Chem., 276: 9583-9586.

Sakai, Y., Koller, A., Rangell, L.K., Keller, G.A., and Subramani, S. 1998. Peroxisome degradation by microautophagy in Pichia pastoris: identification of specific steps and morphological intermediates. J. Cell Biol., 141: 625-636.

Sato, T.K., Darsow, T., and Emr, S.D. 1998. Vam7p, a SNAP-25-like molecule, and Vam3p, a syntaxin homolog, function together in yeast vacuolar protein trafficking. Mol. Cell. Biol., 18: 5308-5319.

Scott, S.V., Baba, M., Ohsumi, Y., and Klionsky, D.J. 1997. Aminopeptidase $\mathrm{I}$ is targeted to the vacuole by a nonclassical vesicular mechanism. J. Cell Biol., 138: 37-44.

Scott, S.V., Guan, J., Hutchins, M.U., Kim, J., and Klionsky, D.J. 2001. Cvt19 is a receptor for the cytoplasm-to-vacuole targeting pathway. Mol. Cell., 7: 1131-1141.

Scott, S.V., Hefner-Gravink, A., Morano, K.A., Noda, T., Ohsumi, Y., and
Klionsky, D.J. 1996. Cytoplasm-to-vacuole targeting and autophagy employ the same machinery to deliver proteins to the yeast vacuole. Proc. Natl. Acad. Sci. USA, 93: 12304-12308.

Scott, S.V., Nice, D.C., III, Nau, J.J., Weisman, L.S., Kamada, Y., KeizerGunnink, I., Funakoshi, T., Veenhuis, M., Ohsumi, Y., and Klionsky, D.J. 2000. Apg13p and Vac8p are part of a complex of phosphoproteins that are required for cytoplasm to vacuole targeting. J. Biol. Chem., 275: 25840-25849.

Shintani, T., Mizushima, N., Ogawa, Y., Matsuura, A., Noda, T., and Ohsumi, Y. 1999. Apg10p, a novel protein-conjugating enzyme essential for autophagy in yeast. EMBO J., 18: 5234-5241.

Shintani, T., Suzuki, K., Kamada, Y., Noda, T., and Ohsumi, Y. 2001. Apg2p functions in autophagosome formation on the perivacuolar structure. J. Biol. Chem., 276: 30452-30460.

Straub, M., Bredschneider, M., and Thumm, M. 1997. AUT3, a serine/ threonine kinase gene, is essential for autophagocytosis in Saccharomyces cerevisiae. J. Bacteriol., 179: 3875-3883.

Stromhaug, P.E., and Klionsky, D.J. 2001. Approaching the molecular mechanism of autophagy. Traffic, 2: 524-531.

Suriapranata, I., Epple, U.D., Bernreuther, D., Bredschneider, M., Sovarasteanu, K., and Thumm, M. 2000. The breakdown of autophagic vesicles inside the vacuole depends on Aut4p. J. Cell Sci., 113: 40254033.

Suzuki, K., Kirisako, T., Kamada, Y., Mizushima, N., Noda, T., and Ohsumi, Y. 2001. The pre-autophagosomal structure organized by concerted functions of $A P G$ genes is essential for autophagosome formation. EMBO J., 20: 5971-5981.

Tanida, I., Mizushima, N., Kiyooka, M., Ohsumi, M., Ueno, T., Ohsumi, Y., and Kominami, E. 1999. Apg7p/Cvt2p: A novel protein-activating enzyme essential for autophagy. Mol. Biol. Cell, 10: 1367-1379.

Tanida, I., Tanida-Miyake, E., Komatsu, M., Ueno, T., and Kominami, E. 2002. Human Apg3p/Aut1p homologue is an authentic E2 enzyme for multiple substrates, GATE-16, GABARAP, and MAP-LC3, and facilitates the conjugation of hApg12p to hApg5p. J. Biol. Chem., 277: 13739-13744.

Tanida, I., Tanida-Miyake, E., Ueno, T., and Kominami, E. 2001. The human homolog of Saccharomyces cerevisiae Apg7p is a proteinactivating enzyme for multiple substrates including human Apg12p, GATE-16, GABARAP, and MAP-LC3. J. Biol. Chem., 276: 17011706.

Teter, S.A., Eggerton, K.P., Scott, S.V., Kim, J., Fischer, A.M., and Klionsky, D.J. 2001. Degradation of lipid vesicles in the yeast vacuole requires function of Cvt17, a putative lipase. J. Biol. Chem., 276: 20832087.

Teter, S.A. and Klionsky, D.J. 1999. How to get a folded protein across a membrane. Trends Cell Biol., 9: 428-431.

Teter, S.A. and Klionsky, D.J. 2000. Transport of proteins to the yeast vacuole: autophagy, cytoplasm-to-vacuole targeting, and role of the vacuole in degradation. Semin. Cell Dev. Biol., 11: 173-179.

Uetz, P., Giot, L., Cagney, G., Mansfield, T.A., Judson, R.S., Knight, J.R., Lockshon, D., Narayan, V., Srinivasan, M., Pochart, P., Qureshi-Emili, A., Li, Y., Godwin, B., Conover, D., Kalbfleisch, T., Vijayadamodar, G., Yang, M., Johnston, M., Fields, S., and Rothberg, J.M. 2000. A comprehensive analysis of protein-protein interactions in Saccharomyces cerevisiae. Nature, 403: 623-627.

Ulrich, H.D. 2002. Natural substrates of the proteasome and their recognition by the ubiquitin system. Curr .Top. Microbiol. Immunol., 268: 137174.

Voges, D., Zwickl, P., and Baumeister, W. 1999. The 26S proteasome: a molecular machine designed for controlled proteolysis. Annu. Rev. Biochem., 68: 1015-1068.

Wang, Y.X., Catlett, N.L., and Weisman, L.S. 1998. Vac8p, a vacuolar protein with armadillo repeats, functions in both vacuole inheritance and 
protein targeting from the cytoplasm to vacuole. J. Cell Biol., 140: 1063-1074.

Wang, H., Bedford, F.K., Brandon, N.J., Moss, S.J., and Olsen, R.W. 1999. $\mathrm{GABA}_{\mathrm{A}}$-receptor-associated protein links $\mathrm{GABA}_{\mathrm{A}}$ receptors and the cytoskeleton. Nature, 397: 69-72.

Wang, C.-W., Kim, J., Huang, W.-P., Abeliovich, H., Stromhaug, P.E., Dunn, W.A., Jr., and Klionsky, D.J. 2001. Apg2 is a novel protein required for the cytoplasm to vacuole targeting, autophagy, and pexophagy pathways. J. Biol. Chem., 276: 30442-30451.

Wang, C.-W., Stromhaug, P.E., Shima, J., and Klionsky, D.J. 2002. The Ccz1-Mon1 protein complex is required for the late step of multiple vacuole delivery pathways. J. Biol. Chem., 277: 47917-47927.

Wendland, B., Emr, S.D., and Riezman, H. 1998. Protein traffic in the yeast endocytic and vacuolar protein sorting pathways. Curr. Opin. Cell Biol., 10: 513-522.
Wickner, W. and Haas, A. 2000. Yeast homotypic vacuole fusion: a window on organelle trafficking mechanisms. Annu. Rev. Biochem., 69: 247-275.

Wurmser, A.E. and Emr, S.D. 2002. Novel PtdIns(3)P-binding protein Etf1 functions as an effector of the Vps34 PtdIns 3-kinase in autophagy. J. Cell Biol., 158: 761-772.

Yoshihisa, T. and Anraku, Y. 1990. A novel pathway of import of $\alpha$ mannosidase, a marker enzyme of vacuolar membrane, in Saccharomyces cerevisiae. J. Biol Chem., 265: 22418-22425.

Yuan, W., Tuttle, D.L., Shi, Y.J., Ralph, G.S., and Dunn, W.A., Jr. 1997. Glucose-induced microautophagy in Pichia pastoris requires the $\alpha$ subunit of phosphofructokinase. J. Cell Sci., 110: 1935-1945.

(Received for publication, October 7, 2002

and accepted, October 10, 2002) 